\title{
POSTPONEMENT E OUTROS CONCEITOS APLICADOS À GESTÃO DA PRODUÇÃO: SEMELHANÇAS E DIFERENÇAS
}

\section{THE POSTPONEMENT AND OTHER CONCEPTS APPLIED IN THE PRODUCTION MANAGEMENT: SIMILARITIES AND DIFFERENCES}

\author{
Karine Araújo Ferreira ${ }^{1}$; Rosane Lúcia Chicarelli Alcântara ${ }^{2}$ \\ ${ }^{1}$ Universidade Federal de Ouro Preto - UFOP - João Monlevade - Brasil \\ karine@decea.ufop.br \\ ${ }^{2}$ Universidade Federal de São Carlos - UFSCar - São Carlos - Brasil \\ rosane@dep.ufscar.br
}

\begin{abstract}
Resumo
A estratégia de postponement é cada vez mais utilizada por empresas preocupadas em gerenciar a complexidade e a variedade crescente de produtos. Em sua essência, o postponement consiste em adiar o máximo possível qualquer deslocamento elou configuração final de produtos até que a demanda seja conhecida. Nos últimos anos, o presente tema tem despertado interesse de acadêmicos e executivos em diversos países. Apesar da atenção crescente ao tema, poucos trabalhos abordam as diferenças conceituais e práticas entre postponement e outros conceitos aplicados na gestão da produção, tais como: customização em massa, ponto de desacoplamento, modularidade, estratégias de produção, Just in time e sistemas flexíveis de manufatura. Assim, neste artigo procurar-se-á investigar e definir esses conceitos, apresentando suas semelhanças, diferenças e relacionamento destes com o postponement. A pesquisa realizada neste trabalho é de natureza qualitativa, onde foi realizado um desenvolvimento teórico-conceitual ${ }^{1}$ com base na revisão de literatura realizada neste artigo.
\end{abstract}

Palavras-chave: postponement; customização em massa; ponto de desacoplamento; modularidade; estratégias de produção.

\section{Introdução}

A fim de responder melhor e mais rapidamente as mudanças dos mercados atuais, guiados por consumidores cada vez mais exigentes, com demanda imprevisível, variedade crescente de produtos e curtos ciclos de vida, empresas têm modificado rapidamente seus conceitos estratégicos e operacionais, buscando principalmente a agilidade e a flexibilidade. A adoção do conceito postponement (do inglês, retardo) é identificada como uma importante abordagem para facilitar a realização de conformidade às exigências do cliente devido a sua contribuição para customização dos produtos e serviços (VAN HOEK, 2000a). Na literatura acadêmica, o termo foi originalmente

\footnotetext{
${ }^{1}$ Segundo Miguel (2007), a pesquisa teórico-conceitual envolve modelagens e discussões conceituais que resultam em novas teorias.
} 
introduzido na literatura de marketing por Alderson (1950) que afirma que, em sua essência, o postponement consiste em adiar o máximo possível qualquer movimentação e/ ou configuração final de produtos e serviços no processo produtivo ou de distribuição. Assim, o produto não é deslocado até que a localização da demanda (ou ponto de consumo) seja conhecida, ao mesmo tempo em que sua configuração final só acontece quando as preferências do consumidor são conhecidas.

Nos últimos anos, o presente tema tem despertado interesse de acadêmicos e executivos em diversos países. Trabalhos abordando o conceito podem ser encontrados em diferentes áreas como logística, produção e cadeia de suprimentos (YANG et al., 2004) e em diferentes correntes literárias (gerencial e analítica) (CARDOSO, 2002). Apesar da atenção crescente ao tema, poucos trabalhos abordam as diferenças conceituais e práticas entre postponement e outros conceitos aplicados na gestão da produção, tais como: customização em massa, ponto de desacoplamento, modularidade, estratégias de produção, Just in time e sistemas flexíveis de manufatura. Além disso, a maioria dos trabalhos sobre o assunto apresenta esses conceitos isoladamente. Poucas pesquisas buscam analisálos e discuti-los de maneira abrangente, levando muitos pesquisadores acreditarem erroneamente que o postponement seja sinônimo ou um novo rótulo para esses conceitos. Assim, neste artigo procurar-se-á investigá-los e defini-los, apresentando suas semelhanças, diferenças e relacionamentos com a estratégia de postponement.

A pesquisa realizada neste trabalho é de natureza qualitativa, onde foi elaborado um desenvolvimento teórico-conceitual com base na revisão bibliográfica dos conceitos analisados neste trabalho. Essa revisão permitiu explicar como esses conceitos estão relacionados com o postponement.

Na seção 2, são apresentados os principais conceitos e tipos de postponement identificados na revisão de literatura. As definições sobre customização em massa, ponto de desacoplamento, modularidade, estratégias de produção, Just in time e sistemas flexíveis de manufatura são discutidos na seção 3, assim como sua relação e principais diferenças com a estratégia de postponement. Por fim, são apresentadas as considerações finais do trabalho (seção 4), e em seguida, as referências bibliográficas.

\section{Postponement: definição e tipos}

O conceito de postponement foi introduzido primeiramente na literatura de marketing por Alderson (1950) como uma maneira de mudar a forma, identidade ou lugar de produtos em um momento tão tarde quanto possível dentro dos processos de manufatura e distribuição física. Operacionalmente, isto envolve projetar e desenvolver produtos padrão ou genéricos que possam ser customizados rapidamente e a baixo custo, uma vez que a demanda do consumidor seja 
conhecida. Envolve também a implementação de estratégias de estoque específicas, permitindo produtos em um único local no canal de distribuição até o momento em que o pedido é realizado. Postergar a movimentação do produto foi denominado de "postponement de tempo" (time postponement), enquanto a postergação na diferenciação do produto foi denominada de "postponement de forma" (form postponement).

Em 1965, Bucklin agregou mais detalhes ao trabalho de Alderson, estudou limites de aplicação da estratégia e criou o conceito oposto ao de postponement, o Princípio da Especulação (Principle of Speculation). Esse princípio é o inverso do postponement e consiste em finalizar todas as operações o mais cedo possível no processo de manufatura (BUCKLIN, 1965).

Os trabalhos de Alderson e Bucklin sobre postponement foram visionários para seu tempo, porém os longos lead times (tempo de atravessamento) na produção e na distribuição tornaram difícil a aplicação do conceito e não despertaram a merecida atenção empresarial na época. Depois de 1965 , poucos trabalhos abordaram o assunto e o tema foi retomado no final da década de 80 por Zinn e Bowersox (1988) que propuseram que o postponement poderia ser separado em cinco diferentes tipos, cada qual com uma estrutura de custo própria. Além do postponement de tempo, os autores destacam quatro tipos de postponement de forma, descritos a seguir:

- postponement de etiquetagem: nesta estratégia, os produtos são armazenados sem qualquer rótulo ou etiqueta que identifiquem sua marca. A etiqueta só é afixada uma vez que o produto foi vendido numa marca dentre as diferentes oferecidas pela empresa. Este tipo de postponement é eficaz para empresas que comercializam um produto sob duas ou mais marcas;

- $\quad$ postponement de embalagem: o produto só é embalado após este ter sido vendido em um tamanho, quantidade ou tipo particular de embalagem. Como exemplo, Zinn (1990) destaca os produtos transportados a granel e embalados posteriormente de acordo com especificações do cliente quanto ao tamanho e tipo de embalagens, como os vinhos da Califórnia, engarrafados em diferentes centros de distribuição;

- $\quad$ postponement de montagem: neste caso, não só a operação de embalagem, a própria montagem do produto também é retardada, até que a empresa receba o pedido do cliente. É na etapa de montagem que ocorre a diferenciação dos produtos. Um bom exemplo é dado pela indústria de computadores pessoais, onde o cliente pode ter seu computador montado em uma loja varejista, de acordo com suas especificações de componentes;

- postponement de fabricação: a fabricação somente é concluída após recebimento de um pedido. Produtos semi-elaborados ou mesmo em forma de insumos ficam estocados para que ocorra a diferenciação da mercadoria em um tempo ou local mais próximos da demanda. Como exemplo, Zinn (1990) cita a produção de refrigerantes. Neste caso, faz-se o transporte do xarope (produto semi-acabado) para as unidades engarrafadoras, onde a água e o açúcar só são adicionados 
a bebida final quando a demanda é conhecida.

Para Cooper (1993), o conceito pode ser visto como um determinante da estratégia de distribuição global de uma empresa. Quanto à classificação, o autor especifica quatro possíveis estruturas para as operações finais de manufatura, as quais dependem do tipo de operações finais realizadas, do tipo de produto e do mercado. Três características operacionais básicas foram utilizadas para promover a classificação: marca, formulação e periféricos dos produtos. As quatro estruturas são:

- manufatura centralizada (unicentric postponement) - sistema de manufatura centralizada, em que a fabricação é integrada a uma planta global e a distribuição é feita por encomenda. Adequa-se a casos em que as três características do produto são as mesmas em todos os mercados onde o produto é vendido, ou seja, são padronizadas. Assemelha-se ao postponement de tempo;

- $\quad$ manufatura agrupada (bundled manufacturing) - ideal para produtos com marca global e formulações diferentes em cada mercado, como por exemplo, aparelhos de TV. Nessa alternativa, a diferenciação do produto é retardada no processo produtivo (na fábrica) e o produto semi-elaborado é despachado e mantido em localização centralizada (armazém ou centro de distribuição). Essa estratégia é a combinação do postponement de forma com o de tempo;

- $\quad$ montagem postergada (deferred assembly) - sistema de montagem adiada, em que a produção é puxada e a manufatura final dos produtos acontece nos centros de distribuição locais. Adequado para produtos com uma marca global, com periféricos diferenciados e formulações diferenciadas. Assemelha-se aos postponements de montagem e fabricação descrita anteriormente;

- embalagem postergada (Deferred Packaging) - ideal para quando rótulo e/ou embalagem são diferenciados conforme o mercado. Relaciona-se aos postponements de etiquetagem e embalagem de Zinn e Bowersox (1988).

Em uma perspectiva logística, Bowersox e Closs (1996) definem dois tipos de postponement: o postponement de manufatura (ou forma) e o postponement logístico (de tempo). O postponement de manufatura consiste fabricar um produto base ou padrão em quantidades suficientes para realizar economia de escala, enquanto as características de finalização são adiadas até que os pedidos dos consumidores sejam recebidos. Já o postponement logístico consiste em manter toda linha de produtos já acabados em estoque centralizado. O deslocamento dos estoques é adiado até o recebimento do pedido dos clientes. Quando a demanda ocorre, os pedidos são transportados diretamente ao varejo ou ao consumidor.

Van Hoek (1999) conceitua postponement como uma maneira para atrasar ou postergar o ponto de diferenciação do produto, isto é, atrasar os processos em que os produtos são transformados em especificações únicas para o consumidor. Este mesmo autor define também, além 
do postponement de forma e tempo, o postponement de lugar. Para ele, o postponement de lugar implica em estocar os produtos acabados em localizações centrais, antes da demanda ocorrer (atrasando a determinação do local); enquanto o postponement de tempo consiste em atrasar a movimentação de produtos finais efetuando-a apenas sob pedido (atrasando a determinação de tempo). Percebe-se que para Van Hoek (1999) o postponement logístico engloba o postponement de tempo e o de lugar, ou seja, a combinação de postponement de tempo e lugar é entendida como postponement logístico.

Pagh e Cooper (1998) combinaram quatro estratégias de postponement para a cadeia de suprimentos em uma matriz 2x2 (quadro 1). Na matriz, postponement de forma é chamado de postponement de manufatura (manufacturing postponement) e postponement de tempo é chamado de postponement logístico (logistics postponement). As quatro estratégias são então desenvolvidas pela combinação do postponement de manufatura e logística. As duas primeiras estratégias são representadas tanto pelo postponement de manufatura como pelo postponement logístico. As outras duas são formadas pela combinação de ambas, denominada de estratégia de postponement total, e pela ausência de ambas, denominada de estratégia de especulação completa.

QUADRO 1 - Matriz postponement/especulação e estratégias genéricas da cadeia de suprimentos

\begin{tabular}{|c|c|c|c|}
\hline & & \multicolumn{2}{|c|}{ LOGÍSTICA } \\
\hline & & $\begin{array}{c}\text { Especulação } \\
\text { Estoques descentralizados }\end{array}$ & $\begin{array}{c}\text { Postponement } \\
\text { Estoques centralizados e } \\
\text { distribuição direta }\end{array}$ \\
\hline \multirow{2}{*}{ 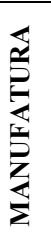 } & $\begin{array}{c}\text { Especulação } \\
\text { Fabricação para } \\
\text { Estocagem } \\
\end{array}$ & $\begin{array}{l}\text { Estratégia de especulação } \\
\text { total }\end{array}$ & $\begin{array}{c}\text { Estratégia de } \\
\text { postponement de logística }\end{array}$ \\
\hline & $\begin{array}{l}\text { Postponement } \\
\text { Fabricação por } \\
\text { Pedidos } \\
\end{array}$ & $\begin{array}{l}\text { Estratégia de postponement } \\
\text { de manufatura }\end{array}$ & $\begin{array}{c}\text { Estratégia de } \\
\text { postponement total }\end{array}$ \\
\hline
\end{tabular}

Fonte: Adaptado de Pagh e Cooper (1998, p.15)

Além destas, várias outras classificações de postponement advindas de diferentes correntes literárias foram elaboradas e mais recentemente, Garcia-Dastugue e Lambert (2007) destacam que o postponement não deve ser implementado sem considerar o impacto para outros membros da cadeia de suprimento, introduzindo o conceito de postponement interorganizacional. Para eles, há situações nas quais múltiplos membros da cadeia de suprimento são capazes de postergar as atividades até que o consumidor final realize o pedido. Adicionalmente, eles diferenciaram os tipos de postponement pela mudança ou não na seqüência de atividades. O postponement pela mudança na seqüência de atividades corresponderia ao postponement de forma e é implementado por mudar o projeto de produto, os processos de manufatura, e a estrutura na rede da cadeia de suprimentos, ou seja, os produtos e os processos são redesenhados usando a padronização, projeto modular ou reestruturação de processos. Por outro lado, o postponement pode ser implementado sem mudar a 
seqüência de atividades, ou seja, sem mudar o projeto do produto, processo de manufatura ou a estrutura da rede da cadeia de suprimentos e é denominado pelo mesmo autor como postponement baseado no tempo. O postponement baseado no tempo se refere ao atraso intencional de atividades ao tempo mais tarde e não requer mudança onde o trabalho é feito; isto é, o ponto de diferenciação do produto não é mudado.

Analisando as várias definições e classificações elaboradas em mais de 50 anos de estudo sobre o tema, é possível afirmar que a sua definição original ainda permanece atual, ou seja, apesar das diferentes conceituações e áreas de aplicação do conceito surgidas no decorrer dos anos, a idéia inicial proposta por Alderson em 1950 não sofreu grandes modificações. As definições mais recentes adicionam a esta definição inicial as possíveis aplicações do postponement, como por exemplo, sua adoção para possibilitar à customização em massa e sua aplicação em toda cadeia de suprimentos. Porém, a idéia inicial proposta por Alderson em 1950, não teve grandes modificações.

\section{Relacionamento entre postponement e outros conceitos à gestão da produção}

Assim como postponement, existem vários outros conceitos que buscam alcançar maior flexibilidade e redução de custo no atendimento às especificações dos clientes. Dentre elas, é possível citar: customização em massa, ponto de desacoplamento do pedido, modularidade, estratégias de produção, just in time (JIT) e sistemas flexíveis de manufatura, entre outras. O objetivo desta seção é apresentar esses conceitos, e explicar o relacionamento existente entre eles e a estratégia de postponement, mostrando suas semelhanças e diferenças.

\subsection{Postponement e customização em massa}

O termo customização em massa (CM) foi originalmente usado por Stan Davis, em seu livro "Futuro Perfeito" de 1987, porém somente tornou-se mais conhecido no meio empresarial, com a publicação do livro "Customização em Massa", por Pine (1993), que define o termo como a produção em massa de bens e serviços que atendam aos anseios específicos de cada cliente individualmente, a custos comparáveis aos dos produtos não customizados. Dessa forma, a proposta da CM é a de oferecer produtos únicos e customizados, em uma escala de produção comparável a da produção em massa, a custos relativamente baixos (GRAMAN e MAGAZINE, 2006).

Assim como a CM, o postponement também possibilita atender os clientes de forma personalizada e a baixo custo. Apesar disso, os dois conceitos não são sinônimos e diferentes autores destacam o relacionamento existente entre eles (FEITIZINGER; LEE, 1997; VAN HOEK et al., 1999a; VAN HOEK et al., 1999b; GRAMAN; MAGAZINE, 2006; YANG et al., 2004b; CAN, 2008; MILLS, 2002). 
Para Feitizinger e Lee (1997), o relacionamento entre postponement e customização em massa pode ser explicado pela seguinte sentença: “a chave para customizar em massa efetivamente é postergar a tarefa de diferenciação de um produto para um consumidor específico até o último ponto possível na rede de suprimentos (companhias de suprimentos, manufatura e de distribuição)". Percebe-se que os mesmos autores consideram o postponement elemento essencial para se obter a customização em massa.

Van Hoek et al. (1999a) afirmam que a customização em massa é uma das estratégias para lidar com a incerteza da demanda e ambientes turbulentos, e o postponement é apresentado como uma estratégia operacional para mover em direção a customização em massa. Em outro artigo, Van Hoek et al.(1999b) destacam que a popularidade do postponement pode ser em parte atribuída pelo crescente interesse na customização em massa, uma vez que o postponement pode fornecer uma solução prática para realizar os benefícios da customização em massa, combinando as estratégias da produção puxada e empurrada em um sistema operacional. Como exemplo, é possível destacar uma cadeia de suprimentos em que à montagem final é executada sob pedido do consumidor (puxado), enquanto as atividades de manufatura à montante são executadas sob previsão (empurradas).

Corroborando com esta idéia, Graman e Magazine (2006) afirmam que um método de conseguir a customização em massa é postergar a tão tarde quanto possível à configuração de produtos padronizados (genéricos) em uma ampla variedade de produtos finais.

O relacionamento entre customização em massa e postponement pode ser também analisado sob o conceito "leagility" que é a combinação de características da cadeia de suprimentos ágil com as potencialidades da cadeia de suprimentos enxuta dentro de uma mesma cadeia de suprimentos. Os paradigmas enxuto (lean) e ágil têm sido muito utilizados no contexto da gestão da cadeia de suprimentos. Ambos têm particularidades distintas e são aplicados em ocasiões específicas. Uma cadeia enxuta foca na qualidade e preço e, ainda, tem como premissa fazer mais com menos. Por sua vez, uma cadeia ágil tem como premissa responder as variações do mercado a fim de atender o cliente adequadamente, podendo melhor responder as variações de volume e às variações nos produtos. (CHRISTOPHER; TOWILL, 2000). Nesse sentido, Can (2008) destaca que customização em massa requer cadeia de suprimentos leagility, porque ambas eficiência e responsividade são importantes princípios para sucesso da estratégia de customização em massa. E o postponement contribui para obtenção de ambos princípios. Dessa forma, pode-se concluir que o postponement contribui para o alcance de uma cadeia de suprimentos leagility, necessária para customização em massa.

Finalmente, a figura 1 proposta por Mills et al. (2002) apresenta as competências e recursos necessários para aplicar a customização em massa, onde o postponement é destacado como apenas um desses recursos. 
FIGURA 1 - Competências necessárias para a customização em massa

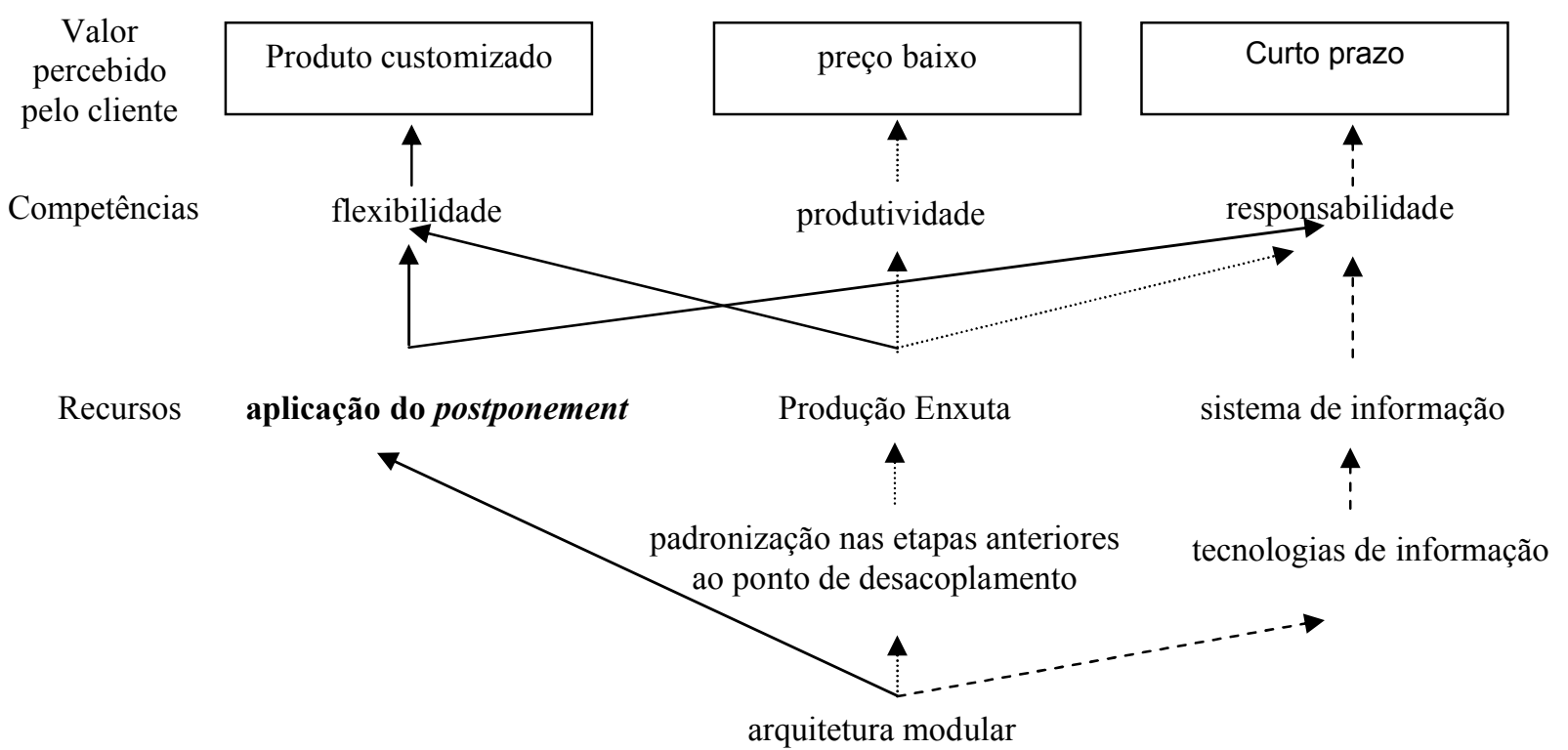

Fonte: Adaptado de Mills et al. (2002)

Baseado nestas considerações, pode-se afirmar que o postponement é apenas um dos possíveis recursos usados para se alcançar os objetivos da customização em massa, que é o de fabricar produtos que atendam as necessidades individuais dos clientes em uma escala comparável a produção em massa, mantendo o baixo custo, ou seja, é a forma de operacionalizar o postponement.

\subsection{Postponement e ponto de desacoplamento}

Um importante conceito ao se abordar o tema postponement é o de Customer Order Decoupling Point (CODP - ponto de desacoplamento do pedido do consumidor), ou simplesmente, ponto de desacoplamento, que pode ser entendido como o ponto do processo de produção até onde o consumidor exerce influência direta na produção. Ou ainda, é o ponto de separação entre o que é produzido para estoque e o que é produzido sob encomenda. Além disso, o CODP especifica a posição na cadeia de suprimentos onde a customização ocorre. Assim, todas as atividades na cadeia de suprimentos executadas depois do CODP são customizadas e etiquetadas no pedido específico do consumidor, enquanto atividades na cadeia de suprimentos executadas antes do CODP são padronizadas. (LAMPEL e MINTZBERG, 1996; VAN DONK, 2001; YANG e BURNS, 2003; PIRES, 2004).

$\mathrm{Na}$ literatura, este ponto pode receber diferentes denominações. Além do já mencionado CODP - Customer Order Decoupling Point, existem autores que o definem apenas como DP (decoupling point - ponto de desacoplamento), ou OPP (order penetration point - ponto de penetração do pedido). Sharman (1984) introduz CODP em um contexto logístico, enfatizando as especificações do produto e estoque. Ele define CODP como o ponto onde especificações de 
produto estão tipicamente inalteradas até que a demanda seja conhecida, e o último ponto no qual o estoque é mantido.

Uma representação de CODP é descrita por Garcia-Dastugue e Lambert (2007) conforme figura 2.

FIGURA 2 - Representação de ponto de desacoplamento do pedido do consumidor (CODP)

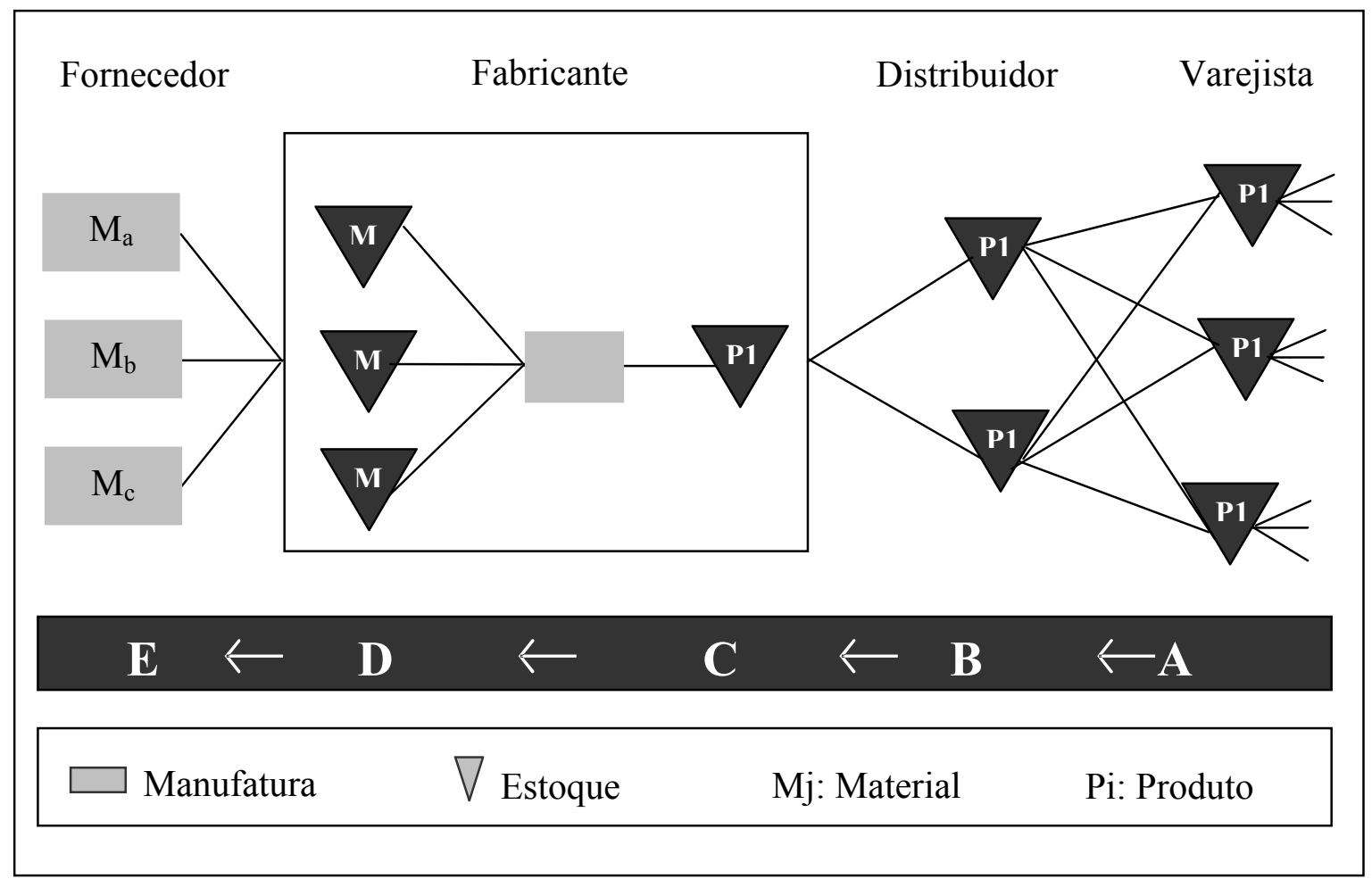

Fonte: Garcia-Dastugue e Lambert (2007, p.62)

A Figura 2 representa uma cadeia de suprimento com quatro camadas potenciais para localizar o CODP: matéria-prima ao produtor, bens acabados ao produtor ou, bens acabados ao distribuidor e bens acabados ao varejista. Mover o estoque de A para B e de B para C é denominado como centralização de estoques. Se as atividades de manufatura podem ser atrasadas, então o estoque ou alguma porção dele deve ser movida de C para D. Neste caso, mudanças na forma ou identidade devem ser atrasadas no tempo sem atrasar o projeto do produto, o projeto do processo de produção, ou a estrutura da rede cadeia de suprimentos. Os estoques, ou alguma porção deles, podem ainda ser movidos de D para E, por exemplo, pela colocação atrasada de pedidos ao fornecedor como sugerido por Bucklin (1965).

Segundo Yang e Burns (2003), duas diferentes perspectivas de CODP são encontradas na literatura. O primeiro é o já referido CODP material que corresponde ao denominado ponto de penetração de ordens (CHRISTOPHER; TOWILL, 2000) e diz respeito à posição dos materiais na cadeia em que o fluxo do produto muda de "empurrado" para "puxado". O segundo ponto é o ponto de desacoplamento da informação. O CODP da informação é abordado por Mason-Jones e Towill 
(1999), que o definem como o ponto na cadeia de suprimentos em que a informação do pedido se torna disponível, sem que haja modificação posterior. É onde os fluxos de informações do mercado e o de previsão de vendas encontram-se. A noção central é que, como o fluxo tradicional de produtos, há vários pontos na cadeia de suprimentos onde a informação é desacoplada e é desconectada entre as funções e as companhias envolvidas.

Os locais ideais em que os pontos de desacoplamento devem ser estabelecidos variam para cada cadeia de suprimentos. Mason-Jones e Towill (1999) recomendam uso de simuladores como ferramenta de pesquisa e entendimento do impacto de decisões sobre o desempenho das cadeias de suprimentos. Embora o ponto de desacoplamento material possa localizar-se em diferentes posições na cadeia de suprimentos para cada estratégia de postponement, o princípio que dirige sua localização é sempre o movimento para a posição mais próxima possível do consumidor, garantindo lead times menores. Já no caso da localização do ponto de desacoplamento da informação, a lógica inverte-se, conforme pode ser visualizado na figura 4. Ele deve ficar no ponto mais próximo possível do início da cadeia, evitando a chegada de informações distorcidas, como o "efeito chicote" ou Forrester.

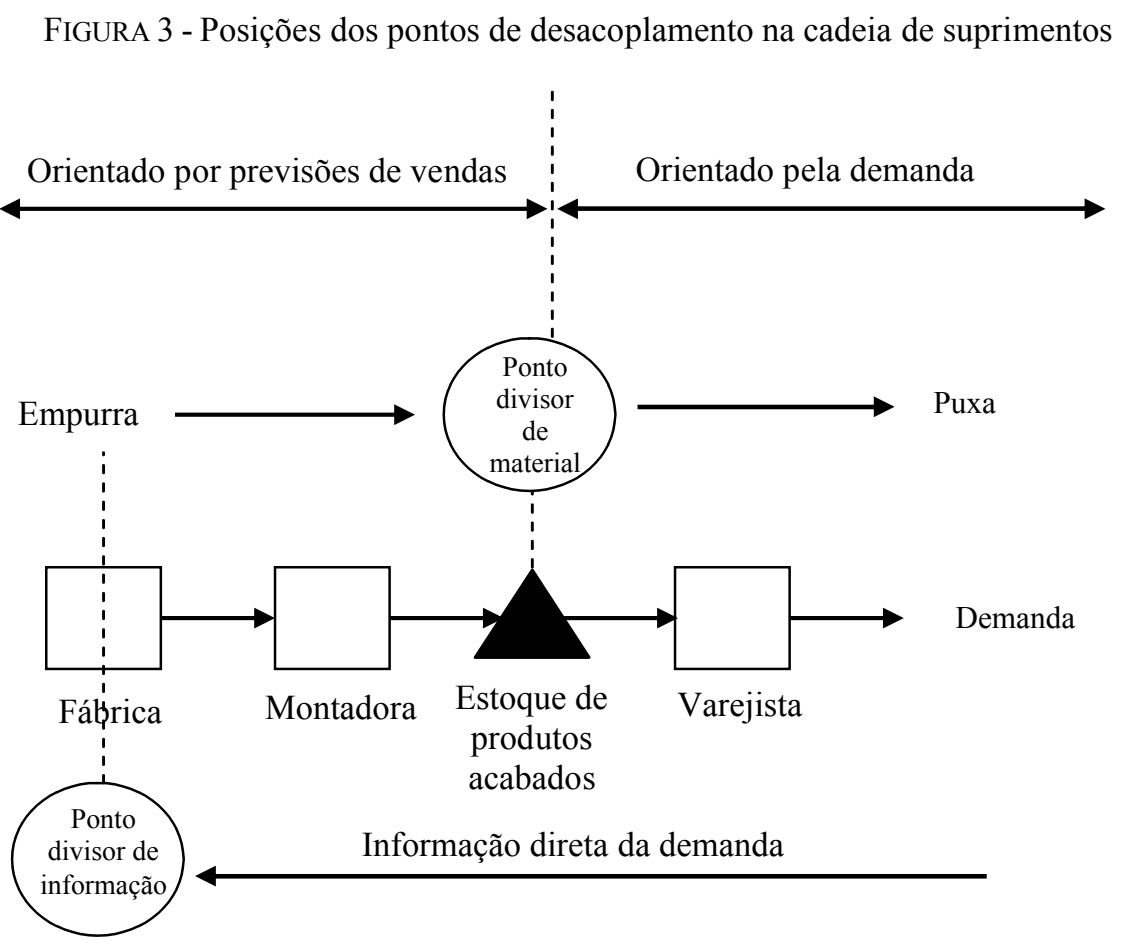

Fonte: Adaptado de Mason-Jones e Towill (1999)

Rudberg e Wikner (2004) afirmam que a posição do CODP depende do balanço de duas forças contrárias: produtividade e flexibilidade. Quando o custo é a principal vantagem competitiva, produtividade força CODP à jusante. Por outro lado, quando flexibilidade e exigências específicas 
do consumidor são os objetivos, flexibilidade empurra a posição do CODP à montante. Esta idéia é ilustrada na figura 4.

FIGURA 4 - O trade off produtividade-flexibilidade e o posicionamento do CODP

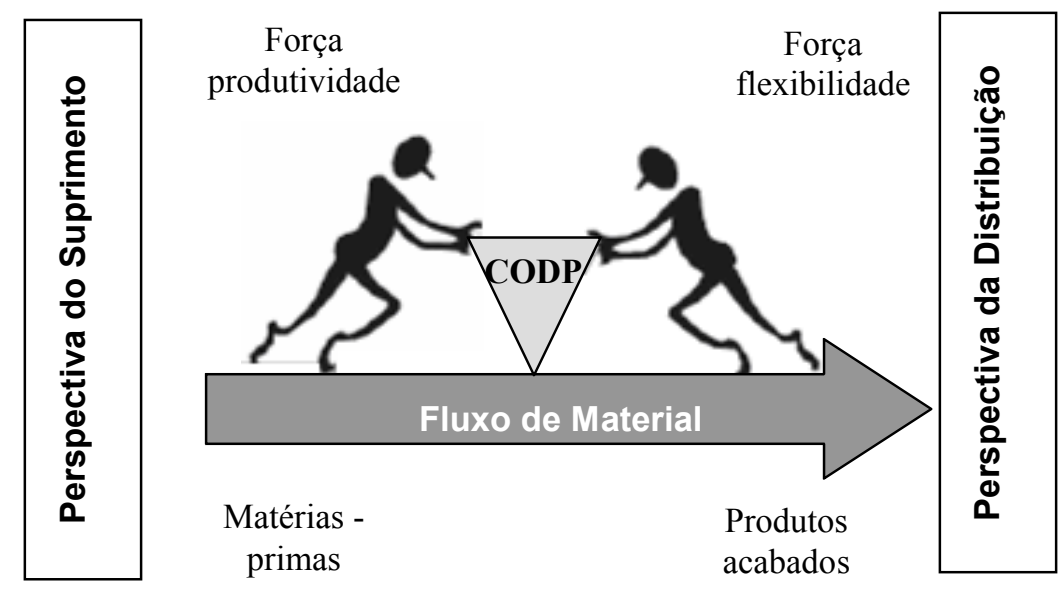

Fonte: Adaptado de Rudberg e Wikner (2004).

Duas outras implicações sobre o ponto de desacoplamento são também discutidas na literatura. Primeiramente, Garcia-Dastugue e Lambert (2007) destacam que múltiplos pontos de desacoplamento podem ser necessários na cadeia de suprimentos. Freqüentemente, na literatura sobre postponement, as cadeias suprimentos são descritas como tendo um único ponto desacoplamento (MASON-JONES; TOWILL 1999; VAN HOEK 2001). Entretanto, Bucklin (1965) sugeriu que mais de um ponto que desacoplamento é requerido em uma cadeia de suprimentos. Em seu trabalho de pesquisa, Garcia-Dastugue e Lambert (2007) mostraram que ter um ponto desacoplamento através da cadeia de suprimentos é uma simplificação conceitual. Corroborando com esta idéia, Yang e Burns (2003) destacam que como muitos dos produtos hoje oferecem variações, opções e características customizadas, há vários pontos de diferenciação do produto que podem ser determinados por múltiplos atributos do produto, objetivos do segmento de mercado e regiões de venda. Por exemplo, produtos de base geral podem ser primeiro diferenciados em famílias e, mais tarde, em produtos finais individuais. Companhias podem simplesmente manter os produtos em o estado não acabado no primeiro ponto de diferenciação do produto até que o pedido do consumidor chegue. Em outras palavras, um pedido do consumidor não tem que ser completamente especificado em um único ponto no tempo. Isto também dá aos consumidores liberdade para especificar certos detalhes do seu pedido e mais tarde, detalhes adicionais.

A segunda implicação se refere ao relacionamento entre ponto de desacoplamento e ponto de diferenciação do produto. Segundo Garg e Tang (1997), ponto de diferenciação do produto refere-se aos estágios nos quais diferentes customizações ocorrem. Para estes autores, ponto de desacoplamento e ponto de diferenciação são vistos como sinônimos. Por outro lado, Garcia- 
Dastugue e Lambert (2007) afirmam que o ponto de desacoplamento é conceitualmente diferente do ponto de diferenciação do produto. Em algumas situações, o ponto de diferenciação do produto e o ponto de desacoplamento podem estar na mesma posição, mas em outros não. Por exemplo, a centralização do estoque consiste consolidar os estoques de múltiplos centros de distribuição em uma posição central, e o ponto de desacoplamento é movido à montante sem mudar a estrutura da cadeia. Neste caso, o ponto de desacoplamento (ou a parte dele) são movidos à montante, enquanto o ponto do diferenciação de produto é inalterado.

A figura 5 proposta por Yang e Burns (2003) exemplifica os vários pontos de desacoplamento possíveis e o seu relacionamento com postponement.

FIGURA 5 - Estratégias de postponement e posicionamento do CODP

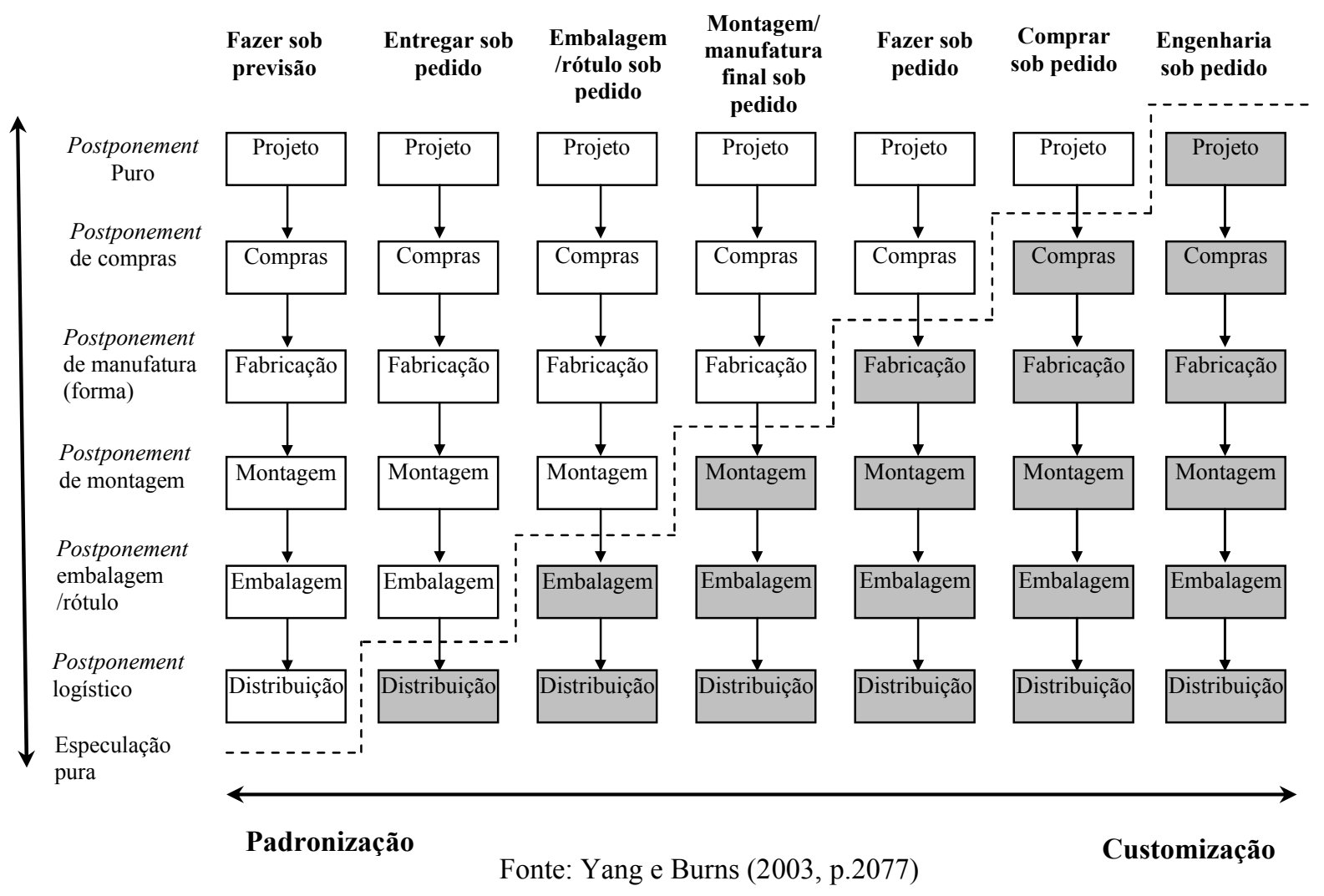

A figura 5 apresenta as diferentes estratégias de postponement, variando do postponement puro até a especulação pura. As linhas pontilhadas separam as atividades feitas sob previsão (destacadas na cor branca) e as orientadas pelos pedidos (cor cinza) e representam o ponto de desacoplamento para cada um dos estágios da cadeia de suprimentos. Da esquerda para direita, a profundidade do postponement aumenta e o CODP se torna mais distante do usuário final. Segundo Can (2008), para desfazer qualquer desentendimento, é necessário enfatizar que postponement não motiva CODP ficar mais distante do consumidor final. É realmente o oposto. A verdade é "postponement é usado para mover o ponto de desacoplamento (CODP) mais perto do consumidor final e aumentar a eficiência e efetividade da cadeia de suprimentos” (YANG; BURNS, 2003). 
Baseado nestas considerações acredita-se que as divergências de opinião entre os estudiosos e pesquisadores sobre o ponto de desacoplamento e ponto de diferenciação são verificadas principalmente porque a maioria dos autores não distingue o ponto de desacoplamento para as diferentes estratégias de postponement (de forma ou de tempo). No postponement de forma, há maior probabilidade dos pontos de desacoplamento e diferenciação estarem situados no mesmo local e por isso serem tratados como sinônimos. Porém, para o postponement de tempo (ou logístico), dificilmente o ponto de diferenciação e ponto de desacoplamento serão os mesmos, uma vez que é a movimentação do produto que é atrasada e não sua configuração final (diferenciação).

\subsection{Postponement e modularidade}

A habilidade de uma empresa utilizar a abordagem do postponement depende em parte do grau de modularidade de seus produtos e processos. No entanto, tais sistemas não são fáceis de serem projetados e operados.

Starr (1965) iniciou o conceito de modularidade na literatura, que consiste em uma abordagem de desenvolvimento de produtos em que o produto deverá ser formado por meio da montagem de um conjunto de partes padronizadas. Nesta abordagem, diferentes combinações de montagem das partes constituintes deverão resultar em diferentes modelos e variações de produtos finais. Isto possibilita o fornecimento de produtos customizados sem a perda dos benefícios da economia de escala. O verdadeiro desafio da modularidade é projetar mecanismos de montagem eficientes e desenvolver produtos que possam ser separados e ter suas partes padronizadas.

Yang et al. (2004 a) definem duas formas de modularidade:

- a modularidade no projeto se refere à definição dos limites do projeto de um produto e de seus componentes de modo que as características e as tarefas de projeto evitem a criação de fortes interdependências entre componentes específicos (módulos) (BALDWIN; CLARK, 1997). Uma arquitetura inteiramente modular significa que uma mudança feita a um componente não exige uma mudança a outros componentes (ULRICH, 1995).

- a modularidade na produção refere o projeto do processo de produção a fim de fazer produtos complicados, projetando e desenvolvendo os módulos em locais diferentes e unindoos para criar um sistema completo (BALDWIN; CLARK, 1997). Esta modularidade divide o processo de produção em sub-processes que podem ser executados simultaneamente ou em uma ordem seqüencial diferente (LEE, 1998). 
Grande parte dos autores que descrevem sobre os temas consideram que a modularidade (no processo de manufatura) é um facilitador, e em alguns casos, um pré-requisito para o postponement (CUNHA, 2002; MELO et al., 2000; LEE, 1998; YANG et al., 2004a; VAN HOEK, 2001).

Cunha (2002) afirma que a modularidade de componentes facilitam a aplicação de estratégias de postponement e, Melo et al. (2000) tratam a modularidade como uma parte integrante e essencial em um dos tipos de postponement (postponement de forma).

Segundo Lee (1998), para habilitar o atraso de algumas partes dos processos, é necessário que o processo seja capaz de ser quebrado em pequenos sub-processos pela modularidade. Adicionalmente, Yang et al.(2004a) também afirmam que a modularidade nos processos é um prérequisito para o postponement.

Van Hoek (2001) também investiga o relacionamento entre postponement e modularidade e destaca dois impactos da modularidade no postponement. O primeiro é que a modularidade habilita a manufatura final rápida a baixo custo de processamento e aumenta a possibilidade de ajustar produtos aos mercados. O segundo é que alta comonalidade de peças abaixa os níveis de estoque e reduz o risco de estoques obsoletos. Entretanto, a modularidade no processo de produção é necessária para o postponement e modularidade no produto facilita o postponement por contribuir para o desempenho de custos e reduzir o risco de obsolescência.

A tabela 1 apresenta a síntese das considerações apresentadas anteriormente sobre o relacionamento entre postponement e modularidade, de acordo com cada autor.

TABELA 1 - Relacionamento entre postponement e modularidade

\begin{tabular}{|c|c|c|}
\hline Relacionamento & Autor & Teoria \\
\hline \multirow{4}{*}{$\begin{array}{l}\text { Postponement } \\
\text { e } \\
\text { Modularidade }\end{array}$} & $\begin{array}{l}\text { Cunha (2002); } \\
\text { Melo et al.(2000) }\end{array}$ & $\begin{array}{l}\text { Modularidade é parte integrante e essencial do } \\
\text { postponement de forma. }\end{array}$ \\
\hline & $\begin{array}{c}\text { Yang et al. (2004a); } \\
\text { Lee (1998) }\end{array}$ & $\begin{array}{l}\text { Modularidade em processo de produção é um pré- } \\
\text { requisito para o postponement. }\end{array}$ \\
\hline & \multirow[b]{2}{*}{ Van Hoek (2001) } & $\begin{array}{l}\text { Modularidade no processo é necessária para } \\
\text { postponement. }\end{array}$ \\
\hline & & $\begin{array}{l}\text { Modularidade no produto facilita o postponement por } \\
\text { contribuir para o desempenho de custos e reduzir o risco } \\
\text { de obsolescência }\end{array}$ \\
\hline
\end{tabular}

Fonte: Elaborada pela autora

\subsection{Postponement e estratégias de produção}

$\mathrm{Na}$ literatura sobre postponement não existe uma distinção clara sobre as principais diferenças entre este conceito e as estratégias de produção. Alguns autores relacionam os dois conceitos (YANG e BURNS, 2003) e, alguns diferenciam (SKIPWORTH; HARRISON, 2006; SU et al., 2005). 
Antes de apresentar as considerações desses autores, é importante destacar as principais estratégias de produção descritas na literatura. Segundo Pires (2004), há quatro diferentes estratégias de produção:

- $\quad$ Produção para estoque (MTS - Make to Stock): esta estratégia é caracterizada pela produção de bens com base em previsão de demanda (forecast). As vendas são atendidas a partir de estoques pré-existentes de produtos não customizados. Ex: grande parte dos produtos alimentícios.

- Montagem sob encomenda (ATO - Assemble to Order): caracterizada pela manutenção de estoques de subconjuntos, componentes e materiais diversos até o recebimento do pedido do cliente, contendo especificações do produto final. Ex: alguns computadores pessoais.

- $\quad$ Produção sob encomenda (MTO - Make to order): a alternativa do MTO caracterizase pela interação do cliente com o produto, isto é, o produto só é fabricado após recebimento do pedido do cliente. Ex: restaurantes.

- $\quad$ Engenharia sob encomenda (ETO - Engineer to order): é uma extensão do MTO, em que o projeto do produto é elaborado com grande interação com o cliente. É a melhor opção em termos de customização, pois oferece ao cliente, mais liberdade, de escolha. Ex: construção de uma casa.

Além desta classificação apresentada por Pires (2004), outras definições encontradas na literatura destacam também a estratégia BTO (buy to order), ou compras sob pedido (GUNASEKARAN; NGAI, 2005). Está estratégia esta localizada entre as estratégias engenharia sob encomenda (ETO) e produção sob encomenda (MTO). Nela não só a fabricação (como na MTO), mas também a compra de matérias-primas é realizada somente após o recebimento de pedido do cliente.

Para exemplificar essas estratégias, cabe destacar a representação de Yang e Burns (2003), que relacionam os dois conceitos (figura 6 já apresentada anteriormente). Na figura 6 são apresentadas sete estratégias de postponement, variando desde a especulação pura, nos pedidos feitos por previsão da demanda, até o extremo à direita, onde se encontra o postponement puro, caracterizado pela estratégia de engenharia sob pedido (em que todas as atividades desde o projeto do produto até sua entrega só são realizadas depois de pedido firme do cliente). Entre estes extremos estão as atividades de comprar, fabricar, montar, empacotar e entregar sob pedido. A linha pontilhada representa o CODP, conforme já mencionado.

Por outro lado, Skipworth e Harrison (2006) fazem uma distinção entre postponement de forma e as estratégias engenharia sob encomenda (ETO), produção sob encomenda (MTO) e 
produção para estoque (MTS) através da localização do ponto de desacoplamento do pedido do consumidor (CODP). Na situação produção sob encomenda (MTO), o CODP é posicionado antes do início do primeiro processo de transformação, enquanto na situação produção para estoque (MTS), O CODP está localizado depois do final do último processo de transformação. No caso do postponement, o CODP está no estágio do produto semi- acabado (entre MTO e MTS), onde o produto está na forma genérica (figura 6), garantindo que a manufatura final, que diferencia o produto, seja executada sob pedido específico do consumidor. Assim, o postponement de forma reduz o risco associado com a estratégia produção para estoque (MTS) e melhora a responsividadecomparado com engenharia sob pedido (ETO) ou fazer sob pedido (MTO), ao mesmo tempo em que possibilita um alto nível de customização.

FIGURA 6 - Comparação da localização do CODP para postponement e estratégia MTS
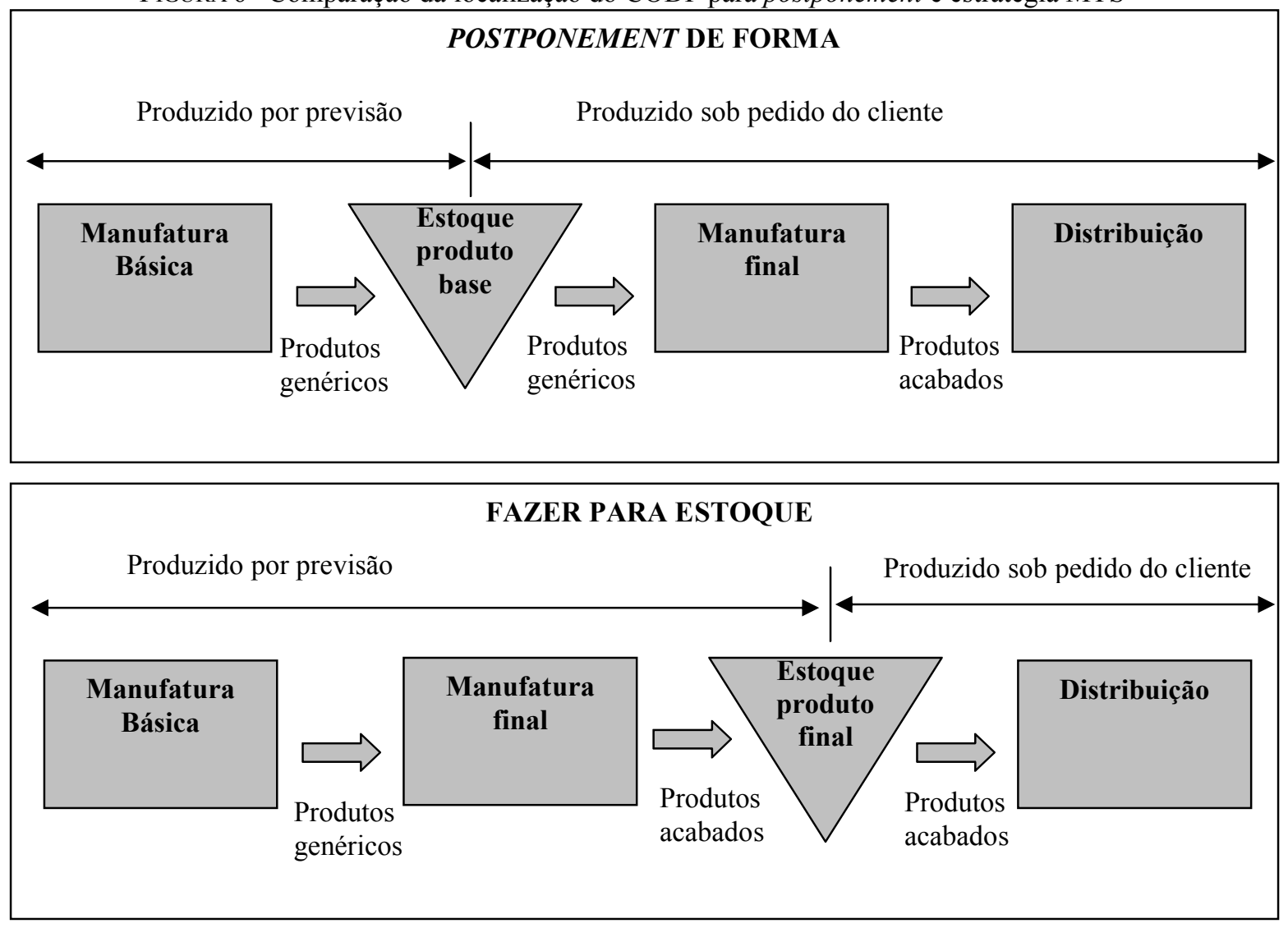

Fonte: Skipworth e Harrison (2006, p.1629)

Su et al. (2005) destacam que aplicar o postponement de forma envolve expedir os produtos em um estado semi-acabado da empresa produtora para uma fábrica à jusante onde ocorra a customização final. Com o objetivo de atrasar a customização final do produto, as firmas estocam um componente genérico (semi-acabado) da qual retiram para a montagem final. Porém, postponement de forma não é necessariamente um processo de montagem sob pedido (ATO).

Em síntese, pode-se verificar que embora alguns autores tratem o postponement e as estratégias de produção com o mesmo significado, os dois conceitos apresentam diferenças. 
Adicionalmente, conforme já mencionado, o postponement pode ocorrer em toda extensão da cadeia de suprimentos, do fornecimento à distribuição final e as atividades podem ser postergadas não somente para próxima etapa do processo produtivo, mas também para outro agente da cadeia de suprimentos. Isto torna a estratégia de postponement muito mais abrangente que a estratégia de produção.

\subsection{Postponement e manufatura JIT/flexível}

A técnica just-in-time (JIT) consiste em produzir bens e serviços exatamente no momento em que são necessários - não antes para que não se transformem em estoque, e não depois para que seus clientes não tenham que esperar. Deve-se produzir aquilo que se vende, na quantidade pedida e no momento e na qualidade indicadas pelos clientes. Além deste elemento temporal do JIT, é possível adicionar a ênfase da técnica na qualidade e eficiência (SLACK et al., 2002). De acordo com Côrrea e Gianesi (1993), o JIT foi desenvolvido no Japão, no meados da década de 70, sendo sua idéia básica e desenvolvimento creditados à fábrica Toyota Motor Company, a qual buscava um sistema de administração que pudesse coordenar a produção com a demanda específica de diferentes modelos e cores de veículos com o mínimo atraso.

Já um sistema de manufatura flexível (flexible manufaturing system - FMS) pode ser definido como uma configuração controlada por computador de estações de trabalho semiindependentes, conectadas por meio de materiais e carregamento de máquinas automatizadas. Essa definição fornece a idéia das partes componentes de um FMS, que são: instalações de carga/descarga, instalações de transporte/manuseio de materiais e, um sistema central de controle de computador. (SLACK et al., 2002).

Embora as estratégias de postponement, just in time (JIT) e manufatura flexível permitam uma companhia obter maior flexibilidade, elas se diferem em vários aspectos, como mostrado no tabela 2 .

TABELA 2 - Relacionamento entre postponement, JIT e sistemas flexíveis de manufatura

\begin{tabular}{l|c|c|c}
\hline & Postponement & Just in Time & $\begin{array}{l}\text { Sistemas flexíveis de } \\
\text { manufatura }\end{array}$ \\
\hline Conceito & Estratégia de atraso & $\begin{array}{c}\text { Sistema de fluxo de } \\
\text { material }\end{array}$ & Automação Flexível \\
\hline $\begin{array}{l}\text { Investimento em sistema } \\
\text { de informação requerido }\end{array}$ & Baixo & Baixo & Alto \\
\hline Alcance de flexibilidade & Várias & Baixo & Alto \\
\hline Exige nível de demanda & Não & Sim & Não \\
\hline $\begin{array}{l}\text { \% de mão de obra } \\
\text { técnica exigida }\end{array}$ & Baixa & Baixa & Alta \\
\hline \begin{tabular}{l} 
Oposto da estratégia \\
\hline
\end{tabular}
\end{tabular}

Fonte: Adaptado de WALLER et al. (2000). 
Muitas companhias têm implementado JIT para reduzir custos de estoque e melhorar qualidade, e estudos têm encontrado evidências desses resultados (CÔRREA e GIANESI, 1993). Usando a filosofia JIT, empresas mantêm somente estoque suficiente para satisfazer a demanda imediata. Segundo Waller et al. (2000), a filosofia JIT pode resultar em postponement em alguns casos, uma vez que usa a abordagem de produção "puxada" (onde a demanda é baseada nos pedidos dos clientes), em oposição à tradicional abordagem de produção "empurrada" (baseada na demanda sob previsão). Por outro lado, é possível usar postponement e não usar JIT. Por exemplo, uma empresa que mantém meses de estoque de produtos semi-acabados ou acabados em seus armazéns, pode estar empregando postponement de manufatura e ou/ logístico, mas não JIT devido à ampla quantidade de estoques. Similarmente, é possível usar JIT sem postponement, como quando produtos são feitos para manter estoque, mas somente em antecipação da demanda diária.

Postponement também difere dos sistemas flexíveis de manufatura (WALLER e CHRISTY, 1992). O último envolve automação programável que permite maior flexibilidade que equipamentos típicos dedicados da maioria dos ambientes de manufatura repetitiva. Embora tais sistemas resultem em mais flexibilidade, eles são extremamente caros e requerem meses ou anos para tornar-se completamente operacionais. Conseqüentemente, sua adoção continua ser lenta. Já o uso do postponement na cadeia de suprimentos pode ser uma alternativa apropriada e de baixo custo.

\section{Considerações finais}

Este artigo apresentou uma discussão sobre como os conceitos customização em massa, ponto de desacoplamento, modularidade, estratégias de produção, Just in time e sistemas flexíveis de manufatura estão relacionados com o postponement.

Inicialmente, cada conceito investigado foi apresentado individualmente, seguido pela análise da relação deste com o conceito de postponement. No quadro 3 a seguir, é apresentada uma síntese dos diversos conceitos discutidos neste artigo e da sua relação com a estratégia de postponement.

O quadro 2 mostra o relacionamento entre postponement e os demais conceitos aplicados à gestão da produção, onde é possível verificar a importância da modularidade e do ponto de desacoplamento para a aplicação do postponement, que por sua vez, é uma ferramenta essencial à adoção da estratégia de customização em massa. Já em relação aos conceitos de postponement, estratégias de produção, Just in time e sistemas flexíveis de manufatura é possível destacar que são conceitos totalmente distintos e independentes, ou seja, a aplicação do postponement não requer a adoção de nenhum desses conceitos e vice-versa.

Examinando esses conceitos, pode-se afirmar que estes são de relevante importância para permitir que as empresas atendam às necessidades individuais de cada cliente de forma 
personalizada e a baixo custo. Apesar disso, eles não são sinônimos e cada um destes apresenta um objetivo definido dentro das empresas. Alguns destes conceitos apresentados podem ser aplicados conjuntamente com o postponement, potencializando aplicação dessa estratégia. Outros são totalmente distintos e independentes do postponement. As características da demanda, nível de flexibilidade, mão-de-obra técnica e objetivos da organização determinarão a escolha de cada conceito e onde devem ser aplicados.

QUADRO 2 - Relacionamento entre postponement e outros conceitos aplicados à gestão da produção

\begin{tabular}{|c|c|}
\hline & POSTPONEMENT \\
\hline $\begin{array}{l}\text { CUSTOMIZAÇÃO } \\
\text { EM MASSA }\end{array}$ & $\begin{array}{l}\text { - postponement é apenas um dos possíveis recursos usados para se alcançar os } \\
\text { objetivos da customização em massa; } \\
\text { - maneira para operacionalizar o postponement. }\end{array}$ \\
\hline $\begin{array}{l}\text { PONTO DE } \\
\text { DESACOPLAMENTO }\end{array}$ & $\begin{array}{l}\text { - o CODP especifica a posição na cadeia de suprimentos em que o postponement } \\
\text { ocorre; } \\
\text { - postponement é usado para mover o CODP para mais perto do usuário final e } \\
\text { aumenta a eficiência e efetividade da cadeia de suprimentos. }\end{array}$ \\
\hline MODULAR & $\begin{array}{l}\text { - modularidade é parte integrante e essencial do postponement de forma. } \\
\text { - modularidade em processo de produção é um pré-requisito para o postponement; } \\
\text { - modularidade no produto facilita o postponement por contribuir para o } \\
\text { desempenho de custos e reduzir o risco de obsolescência. }\end{array}$ \\
\hline $\begin{array}{l}\text { ESTRATÉGIAS DE } \\
\text { PRODUÇÃO }\end{array}$ & $\begin{array}{l}\text { - as diferenças entre postponement e estratégias de produção são dadas pela } \\
\text { localização e posicionamento do CODP; } \\
\text { - na estratégia fazer sob pedido (MTO), o CODP é posicionado antes do início do } \\
\text { primeiro processo de transformação, enquanto na situação fazer para estoque } \\
\text { (MTS), o CODP está localizado depois do final do último processo de } \\
\text { transformação. No caso do postponement, o CODP está no estágio do produto } \\
\text { semi-acabado (entre MTO e MTS), onde o produto está na forma genérica. }\end{array}$ \\
\hline JUST IN TIME & $\begin{array}{l}\text { - o sistema Just in time foca principalmente no fluxo de material; enquanto a idéia } \\
\text { central do postponement é o atraso em certas etapas da produção e /ou logística; } \\
\text { - o postponement não requer o alto nível de demanda e flexibilidade para sua } \\
\text { aplicação, exigidos no Just in time; } \\
\text { - o inverso da estratégia de postponement é a especulação, enquanto no Just in time } \\
\text { é fazer para estoque. }\end{array}$ \\
\hline $\begin{array}{l}\text { SISTEMAS } \\
\text { FLEXÍVEIS DE } \\
\text { MANUFATURA }\end{array}$ & $\begin{array}{l}\text { - os sistemas flexíveis de manufatura focam principalmente na automação flexível. } \\
\text { Já a ênfase do postponement está no atraso em certas etapas da produção e /ou } \\
\text { logística; } \\
\text { - os sistemas flexíveis de manufatura requerem alto investimento em sistema de } \\
\text { informação, flexibilidade e disponibilidade de mão-de-obra. Estes requisitos não } \\
\text { são necessários à aplicação do postponement. }\end{array}$ \\
\hline
\end{tabular}

Fonte: Elaborado pelas autoras. 


\begin{abstract}
The postponement strategy is being increasingly diffused by companies that desire to manage the complexity of the growing variety of products. The postponement involves change the differentiation of goods (form, identity and inventory location) to as late a time as possible, and thus, it could be used to save both in costs related to uncertainty and in the physical movement of the goods. Although the increasing attention to the subject, little is known about the differences of the postponement and other concepts applied in the production management, such as: mass customization, customer order decoupling point, modularization, production strategies, just in time and flexible manufacturing system. The objective of this paper is to understand better these concepts, presenting its similarities, differences and relationship of these with the postponement strategy. This research is qualitative, where a theory-driven empirical research about the concepts analyzed was elaborated.
\end{abstract}

Key-words: postponement; mass customization; customer order decoupling; modularization; production strategies.

\title{
Referências
}

ALDERSON, W. Marketing efficiency and the principle of postponement. Cost and profit outlook, n.3 p. 15-18, 1950.

BALDWIN, C. Y.; CLARK, K. B. Managing in an age of modularity. Harvard Business Review, v.75, n.5, p. 84 - 93 , 1997.

BOWERSOX, D. J.; CLOSS, D. J. Logistical Management: The Integreted Supply Chain Process. New York, NY: McGraw-Hill, 1996.

BUCKLIN, L. P. Postponement, speculation and the structure of distribution channels. Journal of Marketing Research, v.2, p. 26-31, 1965.

cross ${ }^{\text {et }}$

CAN, K. C. Postponement, mass customization, modularization and customer order decoupling point: building the model of relationships. 2008.81p. Master Thesis - Department of Management and Engineering, 0Linkoping University, Institute of Technology, Linköping, Sweden, 2008.

CARDOSO, P. A. O princípio da postergação: um estudo na cadeia de suprimentos das tintas para impressão. 2002. 158p. Tese (Doutorado em Engenharia de Produção) - Departamento de Engenharia Industrial da Pontíficia Universidade Catolica do Rio de Janeiro, Rio de Janeiro, 2002.

CHRISTOPHER, M.; TOWILL, D. R. Supply Chain migration from lean and functional to agile and customized. Supply Chain Management. Supply Chain Management, v.5, n.4, p.206-213, 2000.

cross ref

CORRÊA, H.; GIANESI, I. G.; CAON, M. Just in Time, MRPII e OPT: um enfoque estratégico. São Paulo: Atlas, 1993.

CUNHA, D. C. Avaliação dos resultados da aplicação de postponement em uma grande malharia e confecção de Santa Catarina. 2002. 173 p. Dissertação (Mestrado em Engenharia de Produção) - Departamento de Engenharia de Produção da Universidade Federal de Santa Catarina, Florianópolis, 2002.

ERNST, R.; KAMRAD, B. Evaluation of supply chain structures through modularization and postponement. European Journal of Operational Research, v.124, p.495-510, 2000.

cross ref

FEITIZINGER, E.; LEE, H. L. Mass customization at Hewlett-Packard: the power of postponement. Harvard Business Review, v.75, n.1, p.116-121, 1997.

GARCIA-DASTUGUE, S.; LAMBERT, D. Interorganizational time-based postponement in the supply chain. Journal of Business Logistics, v.28, n.1, p.57-76, 2007.

GARG, A.; TANG, C. S. On postponement strategies for product families with multiple points of differentiation. IIE Transactions, n. 29, p.641-650, 1997. 
GRAMAN, G. A.; MAGAZINE, M. J. Implementation issues influencing the decision to adopt postponement. International Journal of Operations \& Production Management, v.26, n.10, p.1068-1083, 2006.

cross ref

GUNASEKARAN, A.; NGAI, E. W. Build to order supply chain management: a literature review and framework for development. Journal of Operations Management, v.23, n.5, p.423-451, 2005.

cross

LAMPEL, J.; MINTZBERG, H. Customizing customization. Sloan Management Review. v.38, n.1, p.21-30, 1996.

LEE, H. L. Postponement for mass customization: satisfying customer demands for tailor-made products. In: GATTORNA, J. (Ed.). Strategic Supply Chain Alignment. England: Gower Publishing Ltda, 1998. p.77-91.

MASON-JONES, R.; TOWILL, D. R. Using the information decoupling point to improve supply chain performance. The International Journal of Logistics Management, v. 10, n.2, p. 13-26, 1999.

cross ${ }^{\text {ref }}$

MELO, H. L.; EULALIA, L. A. S.; BREMER, C. F. Postponement: uma prática de supply chain management para possibilitar a customização em massa. In: SIMPÓSIO DE ENGENHARIA DE PRODUÇÃO, 7., 2000, Bauru. Anais eletrônicos... Disponível em: <http://www.bauru.unesp.br/acontece/simpep.html>. Acesso em: dez. 2006.

MIGUEL, P. A. C. Estudo de caso na engenharia de produção: estruturação e recomendações para sua condução. Produção (São Paulo), v. 17, p. 216-229, 2007.

MILlS, J.; PLATTS, K.; BOURNE, M. Competing through competences. Cambridge: Cambridge University Press, 2002.

cross'

PAGH, J. D.; COOPER, M. C. Postponement and speculation strategies: how to choose the right strategy. Journal of Business Logistics, v.19, n.2, p.13-32, 1998.

PINE, J. Mass customizing products and services. Planning Review, v.21, n.4, p. 6 - 13, 1993.

PIRES, S. R. I. Gestão da cadeia de suprimentos: conceitos, estratégias, práticas e casos - supply chain management. São Paulo: Atlas, 2004.

RUDBERG, M.; WIKNER, J. Mass customization in terms of the customer order decoupling point. Production Planning \& Control, v.15, n.4, p.445-458, 2004.

cross ${ }^{\text {ref }}$

SHARMAN, G. The Rediscovery of Logistics. Harvard Business Review, v.62, p.71-80, 1984.

STARR, M. K. Modular-production: a new concept. Harvard Business Review, v.43, n.6, p. 131-142, 1965.

SKIPWORTH, H.; HARRISON, A. Implications of form postponement to manufacturing a customized product. International Journal of Production Research, v.44, n.8, p.1627-1652, 2006.

cross ${ }^{\text {ref }}$

SU, J. C.; CHANG, Y.; FERGUSON, M. Evaluation of postponement structures to accommodate mass customization. Journal of Operations Management, v. 23, n.3-4, p.305-318, 2005.

cross ref

VAN DONK, D. P. Make to stock or make to order: the decoupling point in the food processing industries. International Journal Production Economics, v. 69, p. 297-306, 2001.

cross ref

VAN HOEK, R. I. The rediscovery of postponement a literature review and directions for research. Journal of Operations Management, v. 19, n. 2, p.161-184, 2001.

cross ref

. The thesis of leagility revisited. International Journal of Agile Management Systems, v.2, n.3, p.196-201, 
VAN HOEK, R. I.; PEELEN, E.; COMANDEUR, H. R. Achieving mass customization through postponement: a study of international changes. Journal of Market Focused Management, v.3, p.353-368,1999a.

crossef

VAN HOEK, R. I.; VOS, B.; COMANDEUR, H. R Reestructuring European supply chains by implementing postponement strategies. Long Range Planning, v.32, n.5, p.505-518, 1999 b.

WALLER, M. A.; CHRISTY, D. P. A competitive incentives for manufacturing flexibility. International Journal of Production Economics, v.12, n.1, p.35-45, 1992.

cross ${ }^{\text {ref }}$

WALlER, M. A.; DABHOLKAR, P. A.; GENTRY, J. J. Postponement, product, customization, and market-oriented supply chain management. Journal of Business Logistics, v.21, n.2, p. 133-156, 2000.

YANG, B.; BURNS, N. D.; BACKHOUSE, C. J. Postponement: review and an integrated framework. International Journal of Operations \& Production Management, v.24, n.5, p.268-487, 2004a.

cross ${ }^{\text {ref }}$

. Management of uncertainty through postponement. International Journal of Production Research. v. 42 , n.6, p.1049-1064, 2004 b.

cross'ref

YANG, B.; BURNS, N. D. Implications of postponement for the supply chain. International Journal of Production Research. v.41, n.9, p.2975-2090, 2003.

cross ${ }^{\text {ref }}$

ZINN, W. O retardamento da montagem final de produtos como estratégia de marketing e distribuição. Revista de Administração de Empresas. v. 4, p. 53-59, 1990.

ZINN, W.; BOWERSOX, D. J. Planning physical distribution with the principle of postponement. Journal of Business Logistics, v. 9, n.2, p.117-136, 1988.

\section{Nome completo: Karine Araújo Ferreira}

Filiação institucional: Universidade Federal de Ouro Preto

Departamento: Instituto de Ciências Exatas e Aplicadas - ICEA

Função ou cargo ocupado: Professor assistente

Endereço completo para correspondência: Rua 37, nº 115 - Loanda - João Monlevade, MG - Caixa Postal: 24 - Cep: 35931-006

Telefones para contato: (31) 3852 - 8709

e-mail:karine@decea.ufop.br

\section{Nome completo: Rosane Lúcia Chicarelli Alcântara}

Filiação institucional: Universidade Federal de São Carlos

Departamento: Departamento de Engenharia de Produção

Função ou cargo ocupado: Professor assistente

Endereço completo para correspondência: Rod. Washington Luís - Km 235 - São Carlos - SP CEP:13565-905

Telefones para contato:(16) 3351.8296 
e-mail:rosane@dep.ufscar.br

Enviado em: 11/02/2010

Aprovado em: 21/02/2011 